\title{
NOMA Empowered Integrated Sensing and Communication
}

\author{
Zhaolin Wang, Graduate Student Member, IEEE, Yuanwei Liu, Senior \\ Member, IEEE, Xidong Mu, Graduate Student Member, IEEE, Zhiguo \\ Ding, Fellow, IEEE, and Octavia A. Dobre, Fellow, IEEE
}

\begin{abstract}
A non-orthogonal multiple access (NOMA) empowered integrated sensing and communication (ISAC) framework is investigated. A dual-functional base station serves multiple communication users employing NOMA, while the superimposed NOMA communication signal is simultaneously exploited for target sensing. A beamforming design problem is formulated to maximize the weighted sum of the communication throughput and the effective sensing power. To solve this problem, an efficient doublelayer penalty-based algorithm is proposed by invoking successive convex approximation. Numerical results show that the proposed NOMA-ISAC outperforms the conventional ISAC in the underloaded regime experiencing highly correlated channels and in the overloaded regime.
\end{abstract}

Index Terms

Beamforming design, integrated sensing and communication (ISAC), non-orthogonal multiple access (NOMA).

\section{INTRODUCTION}

Recently, with the global commercial deployment of the fifth-generation (5G) wireless communication networks, the concepts of beyond $5 \mathrm{G}(\mathrm{B} 5 \mathrm{G})$ and six-generation $(6 \mathrm{G})$ wireless com-

Zhaolin Wang and Yuanwei Liu are with the School of Electronic Engineering and Computer Science, Queen Mary University of London, London E1 4NS, U.K. (e-mail: zhaolin.wang@qmul.ac.uk; yuanwei.liu@qmul.ac.uk).

Xidong Mu is with the School of Artificial Intelligence, Beijing University of Posts and Telecommunications, Beijing 100876, China (e-mail: muxidong@bupt.edu.cn).

Zhiguo Ding is with the School of Electrical and Electronic Engineering, The University of Manchester, Manchester M13 9PL, U.K. (e-mail: zhiguo.ding@ manchester.ac.uk).

Octavia A. Dobre is with the Faculty of Engineering and Applied Science, Memorial University, St. John's, NL A1B 3X5, Canada (e-mail: odobre@mun.ca) 
munication have received heated discussion. In contrast to $5 \mathrm{G}$, it is envisioned that the networks in B5G and 6G will be designed to carry out simultaneously sensing and communication [1]. Toward this trend, integrated sensing and communication (ISAC) [2], in which the radar sensing and wireless communication are integrated to share the same spectrum and infrastructure, is proposed and have received growing attention from both academia [3] and industries [4].

Motivated by the advantages of ISAC like sharing spectrum and reducing cost, there has been a number of research contributions recently [5]-[7]. Nevertheless, we notice that these works do not consider the impact of spatially correlated channels and overloaded regime on the ISAC system, which is more likely to encounter in the future wireless communication networks due to the massive number of connected devices [8]. For the conventional multi-antenna technique employed in [5]-[7], the communication users will suffer from severe inter-user interference when the channels are highly correlated or the system is overloaded because of the limited spatial degrees of freedom (DoFs). Thus, even the requirement of the communication-only system cannot be satisfied. In this case, it is generally impossible to integrate the sensing function into the communication system. As a remedy, non-orthogonal multiple access (NOMA) can multiplex communication users in the power domain and mitigate the inter-user interference by exploiting successive interference cancellation (SIC) [9], which provides extra DoFs. Furthermore, NOMA can benefit ISAC by allowing more users to be served than the conventional multiple access techniques, thus achieving higher spectral efficiency. However, to the best of the authors' knowledge, the application of NOMA in ISAC has not been studied yet, which motivates this work.

In this letter, we propose a NOMA-ISAC framework, in which the transmitted superimposed signal is exploited for communication and sensing simultaneously and SIC is exploited for interuser interference mitigation. We formulate a beamforming design problem for the maximization of the weighted sum of the communication throughput and the effective sensing power, subject to the minimum communication rate and radar-specific constraints. To solve this non-convex problem, we propose a double-layer penalty-based algorithm based on successive convex approximation (SCA). Our numerical results show that when the spatial correlation is high or the system is overloaded, the proposed NOMA-ISAC achieves better performance trade-off than the conventional ISAC. 


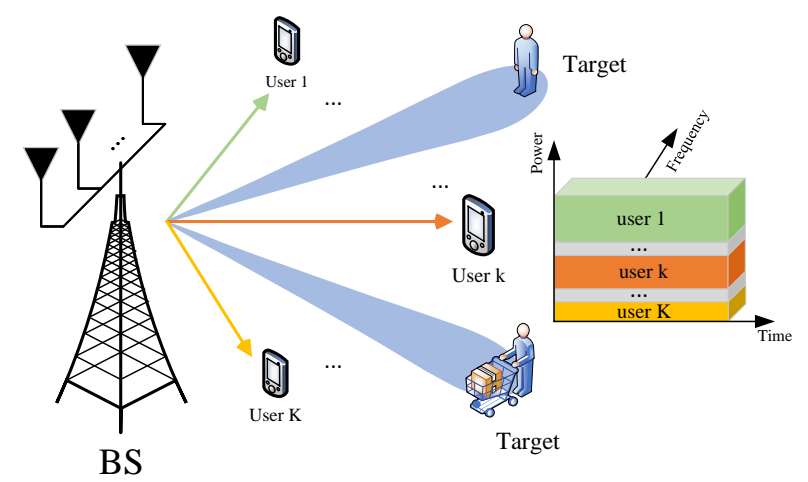

Fig. 1: Illustration of the NOMA-empowered ISAC system.

\section{System Model And Problem Formulation}

\section{A. System Model}

A NOMA-ISAC system is proposed, which consists of a dual-functional base station (BS) equipped with an $N$-antennas uniform linear array (ULA), $K$ single-antenna users indexed by $\mathcal{K}=\{1, \ldots, K\}$, and $M$ radar targets indexed by $\mathcal{M}=\{1, \ldots, M\}$.

1) Communication Model: Different from existing research contributions [5]-[7], in this work, NOMA is employed at the BS for serving multiple communication users [9]. Specifically, the BS transmits the superimposed signals of $\mathbf{w}_{i} s_{i}$ for $\forall i \in \mathcal{K}$ to all users, where $\mathbf{w}_{i} \in \mathbb{C}^{N \times 1}$ are beamformers for delivering the information symbol $s_{i}$ to user $i$. Therefore, the received signal $y_{k}$ at user $k$ is given by

$$
y_{k}=\mathbf{h}_{k}^{H} \sum_{i \in \mathcal{K}} \mathbf{w}_{i} s_{i}+n_{k}=\sum_{i \in \mathcal{K}} \mathbf{h}_{k}^{H} \mathbf{w}_{i} s_{i}+n_{k},
$$

where $\mathbf{h}_{k}=\Lambda_{k}^{-1 / 2} \widetilde{\mathbf{h}}_{k}, \forall k \in \mathcal{K}$ denotes the BS-user channel, $\Lambda_{k}^{-1 / 2}$ and $\widetilde{\mathbf{h}}_{k} \in \mathbb{C}^{N \times 1}$ denote the large and small scale fading, respectively, and $n_{k}$ denotes the circularly symmetric complex Gaussian noise with variance $\sigma_{n}^{2}$. We assume that the users' indexes are in an increasing order with respect to their large-scale channel strength, i.e., $\Lambda_{1}^{-1} \leq \Lambda_{2}^{-1} \leq \cdots \leq \Lambda_{K}^{-1}$. Thus, user 1 is the weakest user while user $K$ is the strongest user. In NOMA, user $k$ first detects and removes the interference from all the weaker $j<k$ users by exploiting SIC, while treating the interference from all the stronger users $j>k$ as noise. Thus, the achievable rate of $s_{k}$ after SIC 
at user $k$ for $\forall k \in \mathcal{K}, k \neq K$ is

$$
R_{k \rightarrow k}=\log _{2}\left(1+\frac{\left|\mathbf{h}_{k}^{H} \mathbf{w}_{k}\right|^{2}}{\sum_{i \in \mathcal{K}, i>k}\left|\mathbf{h}_{k}^{H} \mathbf{w}_{i}\right|^{2}+\sigma_{n}^{2}}\right) .
$$

However, the symbol $s_{k}$ for user $k$ also need to be decodable at user $j$, for $j>k$ and $\forall k \in$ $\mathcal{K}, k \neq K$, to carry out SIC, yielding the following achievable rate

$$
R_{k \rightarrow j}=\log _{2}\left(1+\frac{\left|\mathbf{h}_{j}^{H} \mathbf{w}_{k}\right|^{2}}{\sum_{i \in \mathcal{K}, i>k}\left|\mathbf{h}_{j}^{H} \mathbf{w}_{i}\right|^{2}+\sigma_{n}^{2}}\right) .
$$

Thus, the overall achievable rate of $s_{k}$ for $\forall k \in \mathcal{K}, k \neq K$ is

$$
R_{k}=\min \left\{R_{k \rightarrow k}, \ldots, R_{k \rightarrow K}\right\}
$$

At user $K$, the interference from all the other users is eliminated by SIC. Therefore, its achievable rate is given as

$$
R_{K}=\log _{2}\left(1+\frac{\left|\mathbf{h}_{K}^{H} \mathbf{w}_{K}\right|^{2}}{\sigma_{n}^{2}}\right) .
$$

Therefore, the communication throughput of the $K$ users is given by $R=\sum_{k \in \mathcal{K}} R_{k}$.

2) Sensing Model: In the ISAC system, the communication waveforms can be exploited to perform radar target sensing, but need to satisfy the sensing requirements, which is equivalent to design the covariance matrix of the transmitted signal [10]. The covariance matrix is given by

$$
\mathbf{R}_{\mathbf{w}}=\sum_{i \in \mathcal{K}} \mathbf{w}_{i} \mathbf{w}_{i}^{H}
$$

With the prior information of target, the objective for sensing system is to maximize the effective sensing power, i.e., the power of probing signal in target directions [10], which is given as

$$
P\left(\theta_{m}\right)=\mathbf{a}^{H}\left(\theta_{m}\right) \mathbf{R}_{\mathbf{w}} \mathbf{a}\left(\theta_{m}\right)
$$

where $\theta_{m}, \forall m \in \mathcal{M}$ are target directions and $\mathbf{a}\left(\theta_{m}\right)=\left[1, e^{j \frac{2 \pi}{\lambda} d \sin \left(\theta_{m}\right)}, \ldots, e^{j \frac{2 \pi}{\lambda} d(N-1) \sin \left(\theta_{m}\right)}\right]^{T}$ is the steering vector, where $\lambda$ and $d$ denote the carrier wavelength and antenna spacing, respectively. We assume that similar levels of sensing power is desired in the different target directions such that each target can be fairly tracked. Furthermore, the cross-correlation between transmitted signals at any two target directions $\theta_{k}$ and $\theta_{p}$ is expected to be low such that the sensing system can perform adaptive localization [10]. The cross-correlation is given by $C\left(\theta_{k}, \theta_{p}\right)=$ 
$\left|\mathbf{a}^{H}\left(\theta_{k}\right) \mathbf{R}_{\mathbf{w}} \mathbf{a}\left(\theta_{p}\right)\right|, \forall k \neq p \in \mathcal{M}$. The mean-squared cross-correlation of the $\frac{M^{2}-M}{2}$ pairs of sensing targets is given by

$$
\bar{C}=\frac{2}{M^{2}-M} \sum_{k=1}^{M-1} \sum_{p=k+1}^{M} C\left(\theta_{k}, \theta_{p}\right)^{2} .
$$

Remark 1. In contrast to the conventional ISAC [5], [6], [11], where the inter-user interference is merely mitigated by exploiting spatial DoFs, the NOMA-ISAC system further employs SIC for mitigating inter-user interference, see (2), (3), and (5). Therefore, when the available spatial DoFs are limited (e.g., the underloaded regime with highly correlated channels and overloaded regime), NOMA provides extra DoFs to guarantee the communication performance, which enables the feasibility of integrating the sensing function. This will be verified via the numerical results in Section IV.

\section{B. Problem Formulation}

Given our NOMA-ISAC framework, we aim to maximize the weighted sum of communication throughput and effective sensing power, while satisfying the minimum communication rate of each user and radar-specific requirements. The resultant optimization problem is formulated as

$$
\begin{array}{ll}
\max _{\mathbf{w}_{k}} & \rho_{c} \sum_{k \in \mathcal{K}} R_{k}+\rho_{r} \sum_{m \in \mathcal{M}} P\left(\theta_{m}\right) \\
\text { s.t. } & R_{k} \geq R_{\min , k}, \forall k \in \mathcal{K} \\
& \left|P\left(\theta_{k}\right)-P\left(\theta_{p}\right)\right| \leq P_{\text {diff }}, \forall k \neq p \in \mathcal{M} \\
& \operatorname{diag}\left(\sum_{i \in \mathcal{K}} \mathbf{w}_{i} \mathbf{w}_{i}^{H}\right)=\frac{P_{t} \mathbf{1}^{N \times 1}}{N}, \\
& \bar{C} \leq \xi,
\end{array}
$$

where $\rho_{c} \geq 0$ and $\rho_{r} \geq 0$ are the regularization parameters; by varying them we can obtain the performance trade-off between communication and radar sensing. Here, 9b guarantees the minimum rate of each user and (9c) ensures the similar levels of sensing power in different target directions. The constraint $(9 \mathrm{~d})$ is the constant per antenna constraint [10], where $P_{t}$ denotes the total transmit power. Finally, the constraint $9 \mathrm{e}$ ensures a desired upper bound of the meansquared cross-correlation. However, it is quite challenging to obtain the globally optimal solution for problem (9) due to the following reasons. On the one hand, the expression of achievable 
rate is neither convex nor concave, which makes the objective function non-concave and the constraint $9 \mathrm{~b}$ non-convex. On the other hand, the quadratic form of the covariance matrix makes the constraints $9 \mathrm{c}$ ) and $(9 \mathrm{~d})$ non-convex. In the following, we propose an efficient iterative algorithm to obtain a suboptimal solution by invoking SCA [12].

\section{PROPOSED SOLUTION}

In this section, we develop an SCA-based double-layer iterative algorithm. Firstly, we define $\mathbf{W}_{k} \triangleq \mathbf{w}_{k} \mathbf{w}_{k}^{H}$, which satisfies $\mathbf{W}_{k} \succeq 0, \mathbf{W}_{k}=\mathbf{W}_{k}^{H}$, and $\operatorname{rank}\left(\mathbf{W}_{k}\right)=1$. Then, the problem (9) can be reformulated as

$$
\begin{array}{ll}
\max _{\gamma_{k}, \mathbf{W}_{k}} & f\left(\rho_{c}, \rho_{r}, \gamma_{k}, \mathbf{W}_{k}\right)=\rho_{c} \sum_{k \in \mathcal{K}} \gamma_{k}+\rho_{r} \sum_{m \in \mathcal{M}} P\left(\theta_{m}\right) \\
\text { s.t. } \quad & R_{k} \geq \gamma_{k}, \forall k \in \mathcal{K}, \\
& \gamma_{k} \geq R_{\min , k}, \forall k \in \mathcal{K} \\
& \mathbf{W}_{k} \succeq 0, \mathbf{W}_{k}=\mathbf{W}_{k}^{H}, \forall k \in \mathcal{K} \\
& \operatorname{rank}\left(\mathbf{W}_{k}\right)=1, \forall k \in \mathcal{K} \\
& 9 \mathrm{cc}-9 \mathrm{e} .
\end{array}
$$

Furthermore, We define $\mathbf{H}_{k} \triangleq \mathbf{h}_{k} \mathbf{h}_{k}^{H}$. Then, for $j \geq k$ and $k \in \mathcal{K}, k \neq K$, the constraint (10b) can be rewritten as

$$
R_{k \rightarrow j}=\log _{2}\left(\sigma_{n}^{2}+\sum_{i \in \mathcal{K}, i \geq k} \operatorname{Tr}\left(\mathbf{H}_{j} \mathbf{W}_{i}\right)\right) \underbrace{-\log _{2}\left(\sigma_{n}^{2}+\sum_{i \in \mathcal{K}, i>k} \operatorname{Tr}\left(\mathbf{H}_{j} \mathbf{W}_{i}\right)\right)}_{F_{j, k}} \geq \gamma_{k} .
$$

The non-convexity of this constraint lies in the second term $F_{j, k}$. To address this, we invoke the SCA. By using the first-order Taylor expansion at point $\left(\mathbf{W}_{1}^{n}, \ldots, \mathbf{W}_{K}^{n}\right)$, we have

$$
F_{j, k} \geq \widehat{F}_{j, k} \triangleq-\log _{2}\left(\sigma_{n}^{2}+\sum_{i \in \mathcal{K}, i>k} \operatorname{Tr}\left(\mathbf{H}_{j} \mathbf{W}_{i}^{n}\right)\right)-\frac{\sum_{i \in \mathcal{K}, i>k} \operatorname{Tr}\left(\mathbf{H}_{j}\left(\mathbf{W}_{i}-\mathbf{W}_{i}^{n}\right)\right)}{\left(\sigma_{n}^{2}+\sum_{i \in \mathcal{K}, i>k} \operatorname{Tr}\left(\mathbf{H}_{j} \mathbf{W}_{i}^{n}\right)\right) \ln 2}
$$

Then, we define

$$
\widehat{R}_{k \rightarrow j} \triangleq \log _{2}\left(\sigma_{n}^{2}+\sum_{i \in \mathcal{K}, i \geq k} \operatorname{Tr}\left(\mathbf{H}_{j} \mathbf{W}_{i}\right)\right)+\widehat{F}_{j, k}
$$


which is a lower bound of $R_{k \rightarrow j}$. By exploiting it, the constraint (10b) can be approximated by $\widehat{R}_{k \rightarrow j} \geq \gamma_{k}$. Thus, problem 10 can be reformulated as

$$
\begin{array}{rl}
\max _{\gamma_{k}, \mathbf{W}_{k}} & f\left(\rho_{c}, \rho_{r}, \gamma_{k}, \mathbf{W}_{k}\right) \\
\text { s.t. } & \widehat{R}_{k \rightarrow j} \geq \gamma_{k}, j \geq k, \forall k \in \mathcal{K}, k \neq K, \\
& R_{K} \geq \gamma_{K}, \\
& 9 \mathrm{c}-19 \mathrm{e}, 110 \mathrm{c}-10 \mathrm{e} .
\end{array}
$$

For this optimization problem, the non-convexity is only from the rank-one constraint (10e). Generally, the semidefinite relaxation (SDR) [13] is exploited to solve this problem by omitting the rank-one constraint. Then, the eigenvalues decomposition or Gaussian randomization is used to reconstruct the rank-one solution from the general-rank solution obtained by SDR, which may lead to a significant performance loss and not ensure the feasibility of the reconstructed matrix. To avoid these drawbacks, we attempt to transform the rank-one constraints to a penalty term in the objective function [14], which can also be solved by SCA. Toward this idea, we firstly introduce an equivalent equality constraint:

$$
\left\|\mathbf{W}_{k}\right\|_{*}-\left\|\mathbf{W}_{k}\right\|_{2}=0, k \in \mathcal{K}
$$

where $\|\cdot\|_{*}$ is the nuclear norm, which is the sum of singular values of the matrix, and $\|\cdot\|_{2}$ is the spectral norm, which is the largest singular values of the matrix. Thus, when the matrix $\mathbf{W}_{k}$ is a rank-one matrix, the equality (15) holds. Otherwise, as $\mathbf{W}_{k}$ is semidefinite, we must have that the sum of singular values is larger than the largest singular value, i.e., $\left\|\mathbf{W}_{k}\right\|_{*}-\left\|\mathbf{W}_{k}\right\|_{2}>0$. In order to obtain a rank-one matrix, we introduce a penalty term to the objective function based on (15), yielding

$$
\begin{gathered}
\max _{\gamma_{k}, \mathbf{W}_{k}} f\left(\rho_{c}, \rho_{r}, \gamma_{k}, \mathbf{W}_{k}\right)-\frac{1}{\eta} \sum_{k \in \mathcal{K}}\left(\left\|\mathbf{W}_{k}\right\|_{*}-\left\|\mathbf{W}_{k}\right\|_{2}\right) \\
\text { s.t. } 9 \mathrm{c}-19 \mathrm{e}, 10 \mathrm{c}, 10 \mathrm{~d}, 11 \mathrm{~b}, 11 \mathrm{c}) .
\end{gathered}
$$


However, the second term in the penalty term makes the objective not convex. By exploiting the first-order Taylor expansion at point $\mathbf{W}_{k}^{n}$, its upper bound of is given by

$$
-\left\|\mathbf{W}_{k}\right\|_{2} \leq \widehat{\mathbf{W}}_{k}^{n} \triangleq-\left\|\mathbf{W}_{k}^{n}\right\|_{2}-\operatorname{Tr}\left[\mathbf{v}_{\max , k}^{n}\left(\mathbf{v}_{\max , k}^{n}\right)^{H}\left(\mathbf{W}_{k}-\mathbf{W}_{k}^{n}\right)\right]
$$

where $\mathbf{v}_{\max , k}^{n}$ is the eigenvector corresponding to the largest eigenvalue of $\mathbf{W}_{k}^{n}$. Thus, the problem (16) can be approximated by the following problem

$$
\begin{gathered}
\max _{\gamma_{k}, \mathbf{W}_{k}} f\left(\rho_{c}, \rho_{r}, \gamma_{k}, \mathbf{W}_{k}\right)-\frac{1}{\eta} \sum_{k \in \mathcal{K}}\left(\left\|\mathbf{W}_{k}\right\|_{*}+\widehat{\mathbf{W}}_{k}^{n}\right) \\
\text { s.t. } \quad \text { (9c) }-9 \mathrm{e}, 110 \mathrm{c}, 10 \mathrm{~d}, 114 \mathrm{~b}, 114 \mathrm{c} .
\end{gathered}
$$

The problem (18) is a quadratic semidefinite program (QSDP), which can be efficiently solved by the CVX toolbox [15].

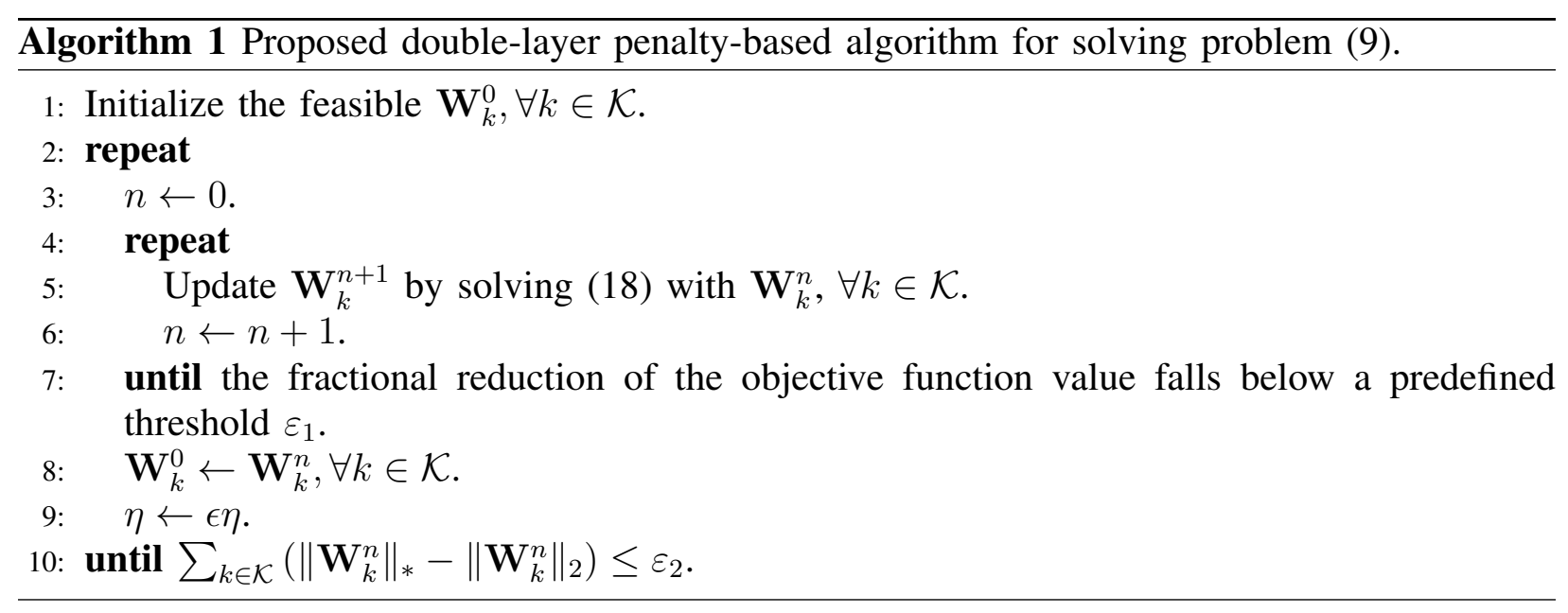

It is worth noting that the choice of parameter $\eta$ plays an important role in the objective function. If this parameter is chosen to be $\eta \rightarrow 0\left(\frac{1}{\eta} \rightarrow \infty\right)$, the rank of matrix $\mathbf{W}_{k}$ will be definitely one. Nevertheless, in this case, we cannot obtain a good solution regarding the maximization of throughput and effective sensing power, since the objective function is dominated by the penalty term. To tackle this, we can initialize a large $\eta$ to obtain a good starting point for the throughput and the effective sensing power. Then, by gradually reducing $\eta$ to a sufficiently small value via $\eta=\epsilon \eta, 0<\epsilon<1$, an overall suboptimal solution can be obtained. This procedure is terminated when the penalty term is sufficiently small, i.e., $\sum_{k \in \mathcal{K}}\left(\left\|\mathbf{W}_{k}\right\|_{*}-\left\|\mathbf{W}_{k}\right\|_{2}\right) \leq \varepsilon_{2}$. The overall algorithm to problem (9) is summarized in Algorithm 1. The complexity of this algorithm is $\mathcal{O}\left(I_{o} I_{i}\left(K^{6.5} N^{6.5} \log (1 / e)\right)\right)$, where $I_{o}$ and $I_{i}$ are the number of iterations of the outer and inner 


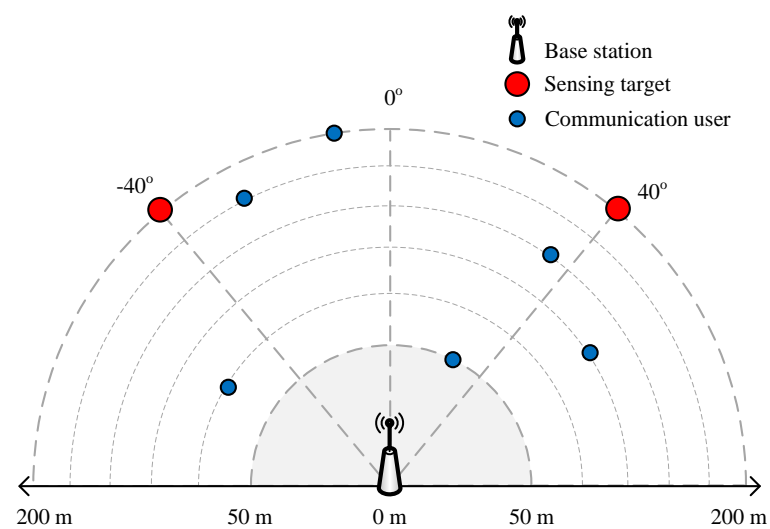

Fig. 2: Simulation setup.

layers, $e$ is the solution accuracy, and $\mathcal{O}\left(K^{6.5} N^{6.5} \log (1 / e)\right)$ is the complexity for solving the QSDP (18) [6].

\section{NUMERiCAL RESULTS}

In this section, the numerical results are provided to demonstrate the characteristics of NOMA in ISAC systems. As shown in Fig. 2, we assume a BS equipped with a ULA with $N=4$ antennas, serving $K=2$ or 6 communication users and tracking $M=2$ radar targets in $\theta_{1}=-40^{\circ}$ and $\theta_{2}=40^{\circ}$. The overall power budget is $P_{t}=20 \mathrm{dBm}$ and the noise power at users is $\sigma_{n}^{2}=-120 \mathrm{dBm}$. The channels between BS and users are assumed to experience Rayleigh fading with the path loss of $\Lambda_{k}(\mathrm{~dB})=32.6+36.7 \log _{10}\left(d_{k}\right)$. In particular, the path loss model is defined based on the 3GPP propagation environment [16, Table B.1.2.1-1]. The fading model follows [17]

$$
\widetilde{\mathbf{H}}=\mathbf{H}_{w} \mathbf{R}_{\widetilde{\mathbf{H}}}^{1 / 2}
$$

where $\widetilde{\mathbf{H}}=\left[\mathbf{h}_{1} /\left\|\mathbf{h}_{1}\right\|, \ldots, \mathbf{h}_{K} /\left\|\mathbf{h}_{K}\right\|\right]$ and $\mathbf{H}_{w}$ is the normalized Rayleigh fading matrix satisfying $\mathbb{E}\left[\mathbf{H}_{w}^{H} \mathbf{H}_{w}\right]=\mathbf{I}$. The matrix $\mathbf{R}_{\widetilde{\mathbf{H}}}$ is the spatial correlation matrix of $\widetilde{\mathbf{H}}$. Its $(i, j)$-th entry indicates the spatial correlation between the channels of user $i$ and user $j$, the norm of which is $t^{|i-j|}$ for $t \in[0,1]$. The users are equally spaced between the distances of $50 \mathrm{~m}$ and $200 \mathrm{~m}$ from BS. We set $R_{\min }=1 \mathrm{bit} / \mathrm{s} / \mathrm{Hz}, P_{\text {diff }}=10$, and $\xi=10$. The initial penalty factor of Algorithm 1 is set to $\eta=10^{5}$. Finally, the convergence thresholds of inner and outer layers are set to $\varepsilon_{1}=10^{-2}$ and $\varepsilon_{2}=10^{-4}$. 


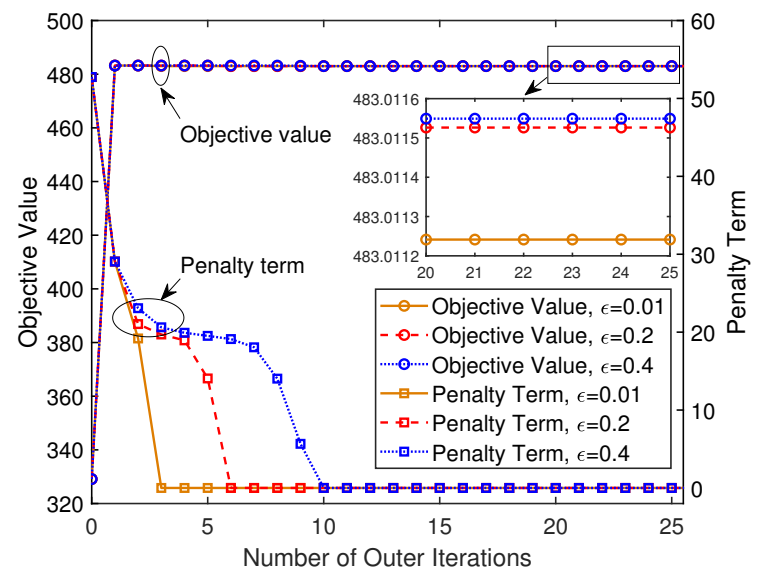

Fig. 3: Convergence of Algorithm 1

\section{A. Convergence of Algorithm 1}

In Fig. 3, the convergence behavior of the proposed algorithm over one random channel realization is studied with $K=6, t=0, \rho_{c}=10$, and $\rho_{r}=1$. We can observe that for any values of the reduction factor $\epsilon$, the objective value quickly converges to a stable value while the penalty term converges to almost zero after several outer iterations. It reveals that the proposed algorithm is capable of finding a feasible rank-one solution with high performance. Furthermore, it can be seen that as the value of $\epsilon$ becomes smaller, the proposed algorithm has higher convergence speed while achieves lower objective value, i.e., worse system performance, which is a trade-off. Thus, in the following simulation, we set $\epsilon=0.2$, which achieves the suitable convergence speed and system performance simultaneously.

\section{B. Baseline}

For comparison, we consider the conventional ISAC system without the employment of NOMA [5], where the achievable rate at user $k$ is given by

$$
R_{k}^{\mathrm{b}}=\log _{2}\left(1+\frac{\left|\mathbf{h}_{k}^{H} \mathbf{w}_{k}\right|^{2}}{\sum_{i \in \mathcal{K}, i \neq k}\left|\mathbf{h}_{k}^{H} \mathbf{w}_{i}\right|^{2}+\sigma_{n}^{2}}\right) .
$$

The corresponding problem of maximizing the throughput $R^{\mathrm{b}}=\sum_{k \in \mathcal{K}} R_{k}^{\mathrm{b}}$ and effective sensing power at the sensing targets can be solved using Algorithm 1 with the interference term in (20). 


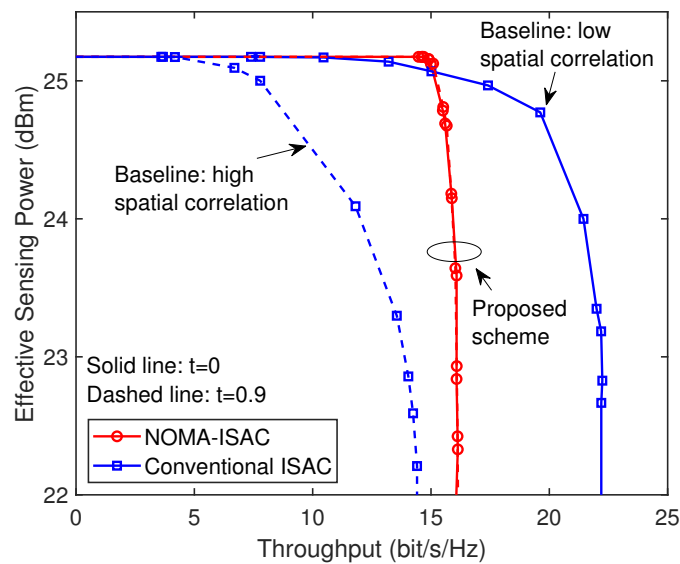

(a) Underloaded $N=4, K=2$

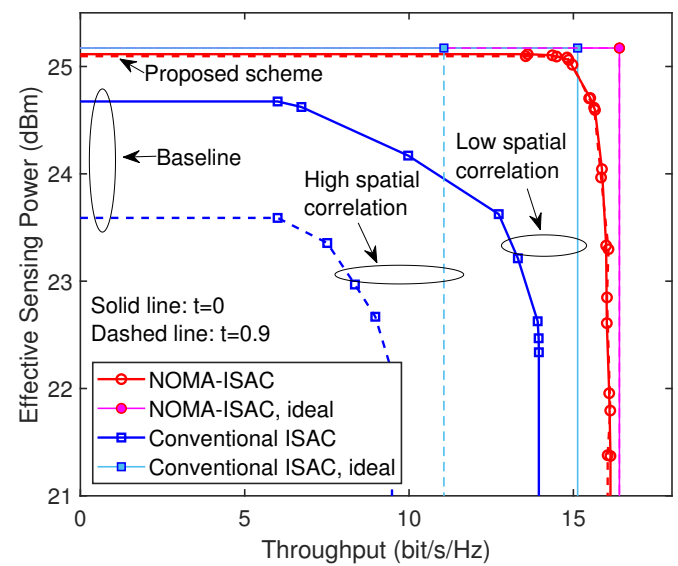

(b) Overloaded $N=4, K=6$

Fig. 4: Trade-off between throughput and effective sensing power.

\section{Performance Trade-off}

In Fig. 4, we demonstrate the performance trade-off, i.e., the communication throughput versus the effective radar sensing power, of NOMA-ISAC and the conventional ISAC. The results are obtained via Monte Carlo simulation over 400 random channel realizations. Two cases are considered, namely the underloaded regime $(K=2)$ and the overloaded regime $(K=6)$. As seen in Fig. 4, in both underloaded and overloaded regimes, the spatial factor has no effect on the NOMA-ISAC system. The reason is that the communication throughput is dominated by the strongest NOMA user. However, the performance of the conventional ISAC system is subject to the spatial factor, i.e., as the spatial correlation increases, the performance achievable area becomes smaller. Specifically, in the underloaded regime (Fig. 4(a) , the NOMAISAC outperforms the conventional ISAC when the spatial correlation is high, but the result is opposite when the spatial correlation is low. In the overloaded regime (Fig. 4(b)], compared to the conventional ISAC, the NOMA-ISAC achieves considerable gain, which becomes even greater when the spatial correlation is high. This is because the inter-user interference cannot be well mitigated in the conventional ISAC system due to the limited spatial DoFs in the overloaded regime. Then, more resources are needed by the conventional ISAC to meet the communication requirements, thus leading to the limited sensing performance. However, for the proposed NOMA-ISAC, although the system is overloaded, the inter-user interference can still be mitigated by SIC, which provides more DoFs to be exploited for the radar sensing. The 


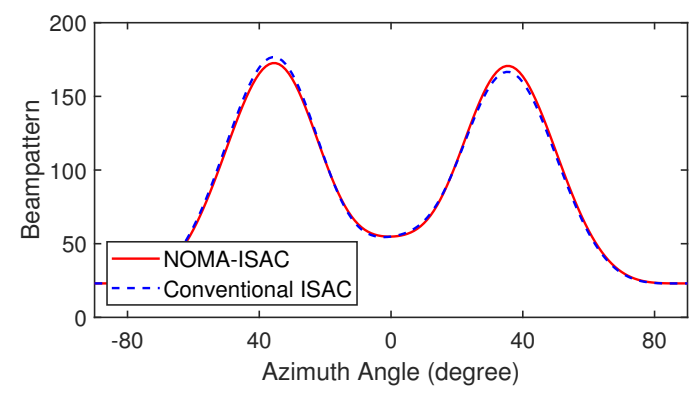

(a) Underloaded $N=4, K=2, t=0$

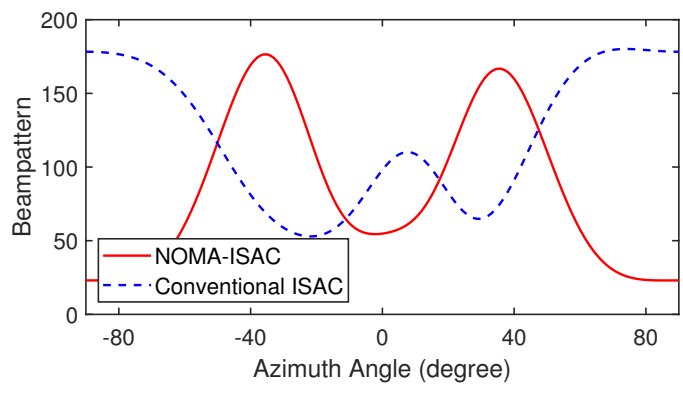

(b) Overloaded $N=4, K=6, t=0$

Fig. 5: Obtained transmit beampattern by different schemes when the communication throughput is $13.5 \mathrm{bit} / \mathrm{s} / \mathrm{Hz}$.

above results underscore the importance of employing NOMA in the ISAC system when the communication system is overloaded or the channels are highly spatially correlated and verify

\section{Remark 1.}

Furthermore, we also demonstrate the ideal ISAC system in Fig. 4(b), i.e., communication and sensing systems work independently, with no effect on each other. The communication throughput and effective sensing power in the ideal ISAC is obtained by removing the communication and sensing from problem (9), respectively. For the conventional ISAC system, it can be seen that there is a significant gap between the real case and the ideal case. However, for the NOMAISAC system, there is only a slight performance gap and the performance upper bounds of communication and radar sensing can be nearly achieved simultaneously.

\section{Transmit Beampattern}

In Fig. 5, we present the obtained transmit beampattern by the proposed NOMA-ISAC and the conventional ISAC over one random channel realization when the communication throughput is $13.5 \mathrm{bit} / \mathrm{s} / \mathrm{Hz}$. We set the spatial correlation factor as $t=0$. Similarly, both underloaded $(K=2)$ and overloaded regimes $(K=6)$ are considered. It can be observed that in the underloaded regime, both NOMA-ISAC and the conventional ISAC can achieve the dominant peak of the transmit beampattern in the directions of interest, i.e., $-40^{\circ}$ and $40^{\circ}$. In the overloaded regime, the dominant peaks can still be achieved by the proposed NOMA-ISAC, while the conventional ISAC experiences severe power leakage in the undesired directions, leading to the significant sensing performance degradation. These results further emphasize the importance of NOMA in 
terms of guaranteeing the sensing performance when the ISAC system is overloaded and also verify Remark 1 .

\section{CONCLUSION}

A NOMA empowered ISAC system has been proposed. A tailor-made beamforming optimization problem was formulated to maximize the weighted sum of communication throughput and effective sensing power subject to the minimum communication rate of each user and the radarspecific requirement. To solve the non-convex problem, a double-layer penalty-based algorithm was developed to obtain a suboptimal solution. Our numerical results indicated that the NOMAISAC system achieves a better communication-sensing trade-off than the conventional ISAC system when the system is overloaded or the channel spatial correlation is high. Furthermore, in the overloaded regime, the performance of the NOMA-ISAC system is close to the ideal ISAC system, which means that in practice, it can provide high quality communication and radar sensing functions simultaneously.

\section{REFERENCES}

[1] K. B. Letaief et al., "The roadmap to 6G: AI empowered wireless networks," IEEE Commun. Mag., vol. 57, no. 8, pp. 84-90, Aug. 2019.

[2] F. Liu et al., "Integrated sensing and communications: Towards dual-functional wireless networks for 6G and beyond," arXiv preprint arXiv:2108.07165, 2021.

[3] J. A. Zhang et al., "Enabling joint communication and radar sensing in mobile networks-a survey," IEEE Commun. Surv. Tut., Early Access, doi: 10.1109/COMST.2021.3122519.

[4] D. K. P. Tan et al., "Integrated sensing and communication in 6G: Motivations, use cases, requirements, challenges and future directions," in Proc. IEEE Int. Online Symp. on Joint Commun. \& Sens. (JC\&S), Feb. 2021, pp. 1-6.

[5] F. Liu et al., "MU-MIMO communications with MIMO radar: From co-existence to joint transmission," IEEE Trans. Wireless Commun., vol. 17, no. 4, pp. 2755-2770, Apr. 2018.

[6] X. Liu et al., "Joint transmit beamforming for multiuser MIMO communications and MIMO radar," IEEE Trans. Signal Process., vol. 68, pp. 3929-3944, Jun. 2020.

[7] F. Dong et al., "Low-complexity beamformer design for joint radar and communications systems," IEEE Commun. Lett., vol. 25, no. 1, pp. 259-263, Jan. 2021.

[8] Y. Liu, S. Zhang et al., "Evolution of NOMA toward next generation multiple access (NGMA)," accepted for the publication in IEEE J. Sel. Areas Commun., https://arxiv.org/abs/2108.04561, 2021.

[9] Y. Liu et al., "Non-orthogonal multiple access for 5G and beyond," Proc. IEEE, vol. 105, no. 12, pp. 2347-2381, Dec. 2017.

[10] P. Stoica et al., "On probing signal design for MIMO radar,” IEEE Trans. Signal Process., vol. 55, no. 8, pp. 4151-4161, Aug. 2007. 
[11] F. Liu et al., "Toward dual-functional radar-communication systems: Optimal waveform design," IEEE Trans. Signal Process., vol. 66, no. 16, pp. 4264-4279, Aug. 2018.

[12] Y. Sun et al., "Majorization-minimization algorithms in signal processing, communications, and machine learning," IEEE Trans. Signal Process., vol. 65, no. 3, pp. 794-816, Feb. 2017.

[13] Z.-Q. Luo et al., "Semidefinite relaxation of quadratic optimization problems," IEEE Signal Process. Mag., vol. 27, no. 3, pp. 20-34, May 2010.

[14] X. Mu et al., "NOMA-aided joint radar and multicast-unicast communication systems," arXiv preprint arXiv:2110.02372, 2021.

[15] M. Grant and S. Boyd, "CVX: Matlab software for disciplined convex programming, version 2.1," http://cvxr.com/cvx, Mar. 2014.

[16] Further Advancements for E-UTRA Physical Layer Aspects (Release 9), document 3GPP TS 36.814, Mar. 2010.

[17] J.-P. Kermoal et al., "A stochastic MIMO radio channel model with experimental validation,” IEEE J. Sel. Areas Commun., vol. 20, no. 6, pp. 1211-1226, Nov. 2002. 